TRANSACTIONS OF THE

AMERICAN MATHEMATICAL SOCIETY

Volume 353, Number 4, Pages 1677-1689

S 0002-9947(00)02650-7

Article electronically published on December 18, 2000

\title{
METRICS AND EMBEDDINGS OF GENERALIZATIONS OF THOMPSON'S GROUP $F$
}

\author{
J. BURILLO, S. CLEARY, AND M. I. STEIN
}

\begin{abstract}
The distance from the origin in the word metric for generalizations $F(p)$ of Thompson's group $F$ is quasi-isometric to the number of carets in the reduced rooted tree diagrams representing the elements of $F(p)$. This interpretation of the metric is used to prove that every $F(p)$ admits a quasiisometric embedding into every $F(q)$, and also to study the behavior of the shift maps under these embeddings.
\end{abstract}

The first example of a finitely presented, infinite, simple group was discovered by R.J. Thompson in 1965. This group $G$ arose in the study of logic and it has many interesting descriptions. In 1974, Higman [10] used a description as the group of automorphisms of the Jónsson-Tarski algebra to generalize $G$ to an infinite family of groups. $G$ has another description as a group of homeomorphisms of the Cantor set. It has subgroups which may be described as homeomorphism groups of different objects, for instance, the circle and the unit interval. Among the people studying these generalizations are Brown [3] and Bieri and Strebel, in a set of unpublished notes [1]. In this paper we will concentrate on the groups $F(p)$, which correspond to the groups $F_{p, \infty}$ in $[3]$. These families of groups have also been extensively studied by Brin and Guzmán [2].

The group $F(2)$, commonly known as Thompson's group $F$, can be regarded as the group of piecewise-linear, orientation-preserving homeomorphisms of the interval $[0,1]$ which have breakpoints only at dyadic points and whose slopes, where defined, are powers of two. In 1984 Brown and Geoghegan 4] found it to be the first example of a finitely presented, infinite-dimensional, torsion-free $F P_{\infty}$ group. This fact has been extended to all $F(p)$ in [3, and studied by Stein [11, where generalizations to more general groups of homeomorphisms with general rational breakpoint sets are considered. Cleary [7] has studied these properties for groups of piecewise-linear homeomorphisms with irrational breakpoints and slopes.

In [5] Burillo established the relationship between the word metric of Thompson's group $F$ and an estimate of the distance derived from the unique normal form of the elements. This algebraic estimate is quasi-isometric to the word metric and was used to prove that some subgroups are nondistorted in $F$. In this paper we find a geometric estimate of the word metric in terms of rooted tree diagrams (see [6]), show that this is quasi-isometric to the word metric, and use this interpretation to prove that some embeddings of $F(p)$ into $F(q)$, including some of those outlined in 2], are quasi-isometric. The interpretations of these embeddings in terms of

Received by the editors September 25, 1998 and, in revised form, August 11, 1999.

2000 Mathematics Subject Classification. Primary 20F65; Secondary 20F05, 20F38, 20E99, $05 \mathrm{C} 25$. 
the tree diagrams also yield insights into the behavior of the shift maps under the embeddings.

After a statement of some results about the groups $F(p)$ in section 1 that will be needed later in the paper, including a brief description of the rooted tree diagrams, section 2 contains the interpretation of the word metric in terms of the normal form and of the number of carets in the tree diagrams. In section 3 we study several embeddings of these groups and show that they are quasi-isometric. The last section is dedicated to studying the behavior of the shift maps of $F(p)$ under these embeddings.

The authors would like to thank the referee for many helpful comments.

\section{The Groups $F(p)$}

The group $F(p)$ is defined by the following infinite presentation:

$$
\left.\mathcal{P}_{p}=\left\langle x_{i}, i \geq 0\right| x_{i}^{-1} x_{j} x_{i}=x_{j+p-1}, \text { for } i<j\right\rangle .
$$

For $p=2$ this group is the well-known Thompson's group $F$. The groups $F(p)$ are natural generalizations of $F$, and share many of its interesting properties. In this section we outline some of these properties that will be used later in the paper. For a very readable introduction to $F$ see [6], and for generalizations to $F(p)$ see [3] and [11.

The groups $F(p)$ admit a shift map $\phi$, which sends every generator $x_{i}$ to $x_{i+1}$. The shift map satisfies $x_{0}^{-1} \phi(x) x_{0}=\phi^{p}(x)$ for all $x \in F(p)$.

The infinite presentation $\mathcal{P}_{p}$ is useful for its symmetry and simplicity, but to discuss the word metric we need to consider a finite presentation. It is clear that $x_{0}, x_{1}, \ldots, x_{p-1}$ generate $F(p)$, and it is possible to write a presentation for $F(p)$ with these $p$ generators and $p(p-1)$ relators (see [9] and [11]). For $p=2$ this is the standard presentation for Thompson's group $F$ :

$$
\left\langle x_{0}, x_{1} \mid\left[x_{0} x_{1}^{-1}, x_{0}^{-1} x_{1} x_{0}\right],\left[x_{0} x_{1}^{-1}, x_{0}^{-2} x_{1} x_{0}^{2}\right]\right\rangle .
$$

In the following, when we refer to the word metric, or the length of an element, we will always mean with respect to these finite presentations.

From the relators $x_{i}^{-1} x_{j} x_{i}=x_{j+p-1}$ we can see that every element in $F(p)$ admits an expression of the form

$$
x_{i_{1}}^{r_{1}} x_{i_{2}}^{r_{2}} \ldots x_{i_{n}}^{r_{n}} x_{j_{m}}^{-s_{m}} \ldots x_{j_{2}}^{-s_{2}} x_{j_{1}}^{-s_{1}}
$$

where $i_{1}<i_{2}<\ldots<i_{n} \neq j_{m}>\ldots>j_{2}>j_{1}$. This expression is unique if we require one additional condition: if both $x_{i}$ and $x_{i}^{-1}$ appear, then one of the generators

$$
x_{i+1}, x_{i+1}^{-1}, x_{i+2}, x_{i+2}^{-1}, \ldots, x_{i+p-1}, x_{i+p-1}^{-1}
$$

must appear as well. This is required for uniqueness because if none of them appears, there is a subword of the type $x_{i} \phi^{p}(x) x_{i}^{-1}$ which can be replaced by $\phi(x)$. This unique expression of an element will be called its normal form. The proof of the uniqueness of the normal form in $F(2)$ in [4] extends easily to every $F(p)$. Given an element $x$, its normal form is the shortest word in the infinite generating set of $\mathcal{P}_{p}$ representing it.

One interpretation for $F(p)$ is based on maps of rooted trees. This interpretation is described with great detail for the case of $F(2)$ in [6]. A rooted $p$-tree is a tree with a distinguished vertex (the root) which has $p$ edges, and any other vertex has 
either valence 1 (leaves) or valence $p+1$ (nodes). We think of a rooted $p$-tree as a descending tree, with the root on top, and different levels of vertices, with the root being the sole vertex of level 0 . Every vertex different from the root is connected by an edge to a vertex in the previous level. Each vertex is either a leaf, in which case it is not connected to the next lower level, or a node with $p$ children, i.e., it is connected to $p$ vertices in the next lower level. A node, together with its $p$ children, and the $p$ edges connecting them, form a $p$-caret. A $p$-caret has, from left to right in the obvious way, a left edge, $p-2$ interior edges, and a right edge.

Following [8, we will say that a caret is a left caret when it is the leftmost caret of a level, or, equivalently, when its vertex is joined to the root by a path made completely out of left edges. We define a right caret in a similar manner, and every caret which is not left or right is called an interior caret.

A $p$-tree diagram will be an order-preserving map between the leaves of two rooted $p$-trees with the same number of carets (and thus the same number of leaves). A diagram is reducible if all the leaves of a caret are sent to all the leaves of another caret in the target. If a diagram is reducible, we will eliminate the two carets whose leaves are mapped to each other. A diagram is called reduced if it is not reducible.

There is a one-to-one correspondence between elements of $F(p)$ and reduced $p$ tree diagrams (see [6] for the case $p=2$ ). See Figure 1 for the reduced tree diagrams which correspond to $x_{0}, x_{1}, \ldots, x_{p-1} \in F(p)$.

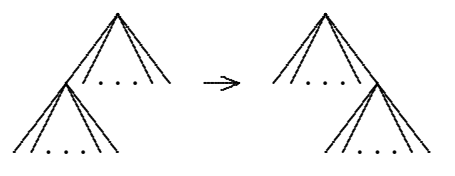

$x_{0}$

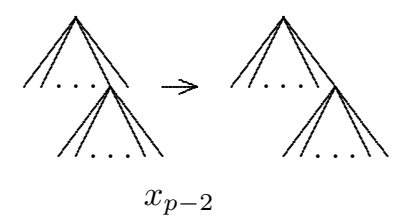

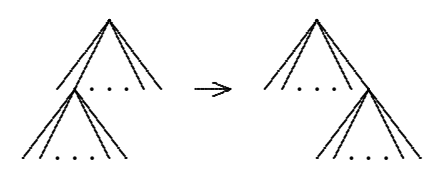

$x_{1}$

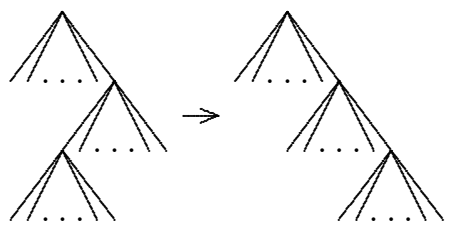

$x_{p-1}$

Figure 1. The tree diagrams for the generators of $F(p)$

The product of two elements $x$ and $y$ can be computed with tree diagrams: Consider the target tree of $x$ and the source tree of $y$. If those trees are equal, then the tree diagram for $x y$ has the source of $x$ as its source and the target of $y$ as its target. If the two trees are not equal, carets must be added to them until the two resulting trees are equal. For every caret added to the target tree of $x$, add a caret to the corresponding leaf in the source tree of $x$. Similarly, add a caret to the target tree of $y$ for each caret added to the source tree of $y$. A tree diagram for $x y$ is obtained by taking the modified source tree of $x$ and the modified target tree of $y$. Reduce if necessary.

See Figure 2 for an example of a product of two tree diagrams. 

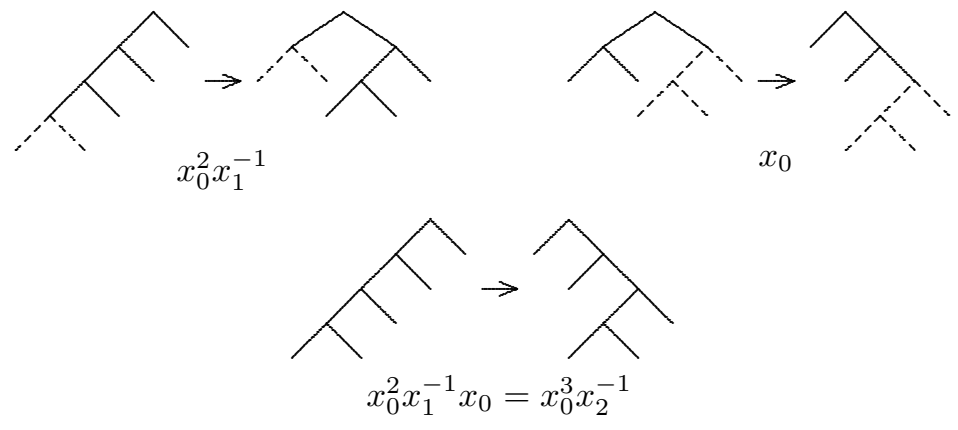

Figure 2. Composing two tree diagrams in $F(2)$, with the added carets in dashes

The set of reduced tree diagrams, with this product, forms a group, and the oneto-one correspondence mentioned above gives an isomorphism between this group of reduced $p$-tree diagrams and $F(p)$. See 6 for the case $p=2$. Fordham, in his unpublished thesis $[\underline{8}$, used this representation to obtain an algorithm to compute the exact word metric for an element of $F(2)$.

The groups $F(p)$ can also be realized as groups of homeomorphisms of the real line $\mathbb{R}$. Each tree will correspond to a subdivision of the interval $[0, \infty)$ in the following manner: the root corresponds to the interval $[0, \infty)$, which gets subdivided into the $p$ intervals $[0,1],[1,2], \ldots,[p-2, p-1]$, and $[p-1, \infty]$. Then every caret subdivides the interval corresponding to its node into $p$ intervals. If the node interval is finite, the $p$ subdivisions have the same length; and if the node interval is of the type $[k, \infty)$, it gets subdivided into $p-1$ intervals of length 1 and one infinite interval. The homeomorphism corresponding to a given tree diagram will be the identity on the interval $(-\infty, 0]$. To construct it on $[0, \infty)$, subdivide the domain according to the source tree and the range according to the target tree. Then interpolate linearly in every interval. For details on this interpretation for $F(2)$, see [4]. We will use both of these geometric representations for $F(p)$ to deduce different properties of these groups.

In a similar manner, the groups $F(p)$ can be interpreted as groups of homeomorphisms of the unit interval. In this case, every caret represents a subdivision of an interval into subintervals of equal length. For a detailed exposition of this interpretation see [6].

Yet another representation of the groups $F(p)$ can be found in [9], in the context of diagram groups. This representation is essentially the same as the tree diagram representation, where a node with its $p+1$ edges is replaced by a 2 -cell with its boundary subdivided in $p+1$ edges, and the cells are attached to each other along the edges according to the same pattern represented by the trees.

\section{The WORD METRIC OF $F(p)$}

The interpretations of the groups $F(p)$, both as groups of homeomorphisms and as groups of maps of rooted trees, can be used to deduce expressions for the word metric. First, we generalize to all $F(p)$ the estimate of the length of an element developed in [5]. This gives a quantity which is equivalent to the length, and can be readily computed from the normal form of an element. Here, we mean equivalent 
in the sense of quasi-isometry. We will denote by $|x|_{p}$ the minimal length of an element of $F(p)$ in the word metric with respect to the generators $x_{0}, x_{1}, \ldots, x_{p-1}$.

Theorem 1. Let $x \in F(p)$ have normal form

and let

$$
x_{i_{1}}^{r_{1}} x_{i_{2}}^{r_{2}} \ldots x_{i_{n}}^{r_{n}} x_{j_{m}}^{-s_{m}} \ldots x_{j_{2}}^{-s_{2}} x_{j_{1}}^{-s_{1}}
$$

$$
D(x)=r_{1}+r_{2}+\ldots+r_{n}+s_{1}+s_{2}+\ldots+s_{m}+i_{n}+j_{m} .
$$

Then, we have

$$
\frac{D(x)}{3(p-1)} \leq|x|_{p} \leq 3 D(x) .
$$

Proof. The upper bound is straightforward: replacing each $x_{i}$ in the normal form by its expression in the generators $x_{0}, x_{1}, \ldots, x_{p-1}$, the length of the word obtained is less than $3 D(x)$, and clearly it is an upper bound for the minimal length.

For the lower bound, observe that the normal form has the shortest possible length among words in the generators of $\mathcal{P}_{p}$ representing $x$. From this we can conclude that $|x|_{p} \geq r_{1}+\ldots+r_{n}+s_{1}+\ldots+s_{m}$. Finally, let $w$ be a word in the generators $x_{0}, x_{1}, \ldots, x_{p-1}$ which has minimal length $|x|_{p}$. To obtain the unique normal form from $w$, the generators have to be switched around using the relators of $\mathcal{P}_{p}$, at the price of increasing the index of one of them by $p-1$ per switch. A given generator in $w$ needs to be switched at most $|x|_{p}-1$ times, so the highest possible generator appearing in the normal form has index at most

$$
p-1+(p-1)\left(|x|_{p}-1\right)=(p-1)|x|_{p}
$$

and then

$$
i_{n} \leq(p-1)|x|_{p} \quad \text { and } \quad j_{m} \leq(p-1)|x|_{p} .
$$

Combining all these inequalities we obtain the desired lower bound.

Part of the previous proof is due to V. Guba, who improved on the proof given in 5.

The number $D(x)$ given in the previous result is then equivalent to the distance. This readily computable quasi-metric $D(x)$ can be used in the place of the genuine word metric to obtain geometric characterizations for the distance.

Proposition 2. Let $x \in F(2)$ be an element whose normal form is a positive word, i.e.,

$$
x=x_{i_{1}}^{r_{1}} x_{i_{2}}^{r_{2}} \ldots x_{i_{n}}^{r_{n}}
$$

Let

$$
N(x)=\max \left\{i_{k}+r_{k}+r_{k+1}+\ldots+r_{n}+1, \text { for } k=1,2, \ldots, n\right\}
$$

Then,

(1) $N(x)$ is equal to the number of carets in either tree of the reduced tree diagram for $x$,

(2) $N(x)$ is equal to the $y$-coordinate of the last breakpoint of the graph of $x$ represented as a homeomorphism of $\mathbb{R}$,

(3) $N(x)$ is quasi-equivalent to the distance. In particular,

$$
\frac{D(x)}{2} \leq N(x) \leq D(x)+1
$$


Proof. The statement that $N(x)$ is equivalent to the distance is clear from the definition: clearly $N(x) \leq D(x)+1$, and also

$$
N(x) \geq i_{1}+r_{1}+\ldots+r_{n}+1 \geq r_{1}+\ldots+r_{n},
$$

and

$$
N(x) \geq i_{n}+r_{n}+1 \geq i_{n}
$$

from which the lower bound follows.

A crucial ingredient for the proofs of (1) and (2) is Theorem 2.5 of [6], which relates the unique normal form directly to the tree diagram.

The proof of statement (1) will proceed by induction on $n$. The case $n=1$ is clear: a computation reveals that the number of carets in either tree of the tree diagram of the element $x_{i}^{r}$ is exactly $i+r+1$.

For the general case, concentrate on the source tree (by Theorem 2.5 of [6] the target tree is composed only of right carets). Let $x=x_{i_{1}}^{r_{1}} x_{i_{2}}^{r_{2}} \ldots x_{i_{n}}^{r_{n}}$ and let $y=x x_{i}^{r}$. Label the leaves of the source tree for $x$ from 0 to $N(x)$ from left to right. To obtain the source tree for $y$ from the tree of $x$, we would have to attach extra carets. If $i \leq N(x)$, attach $r$ carets to the leaf labelled $i$, each hanging from the left child of the caret above. Then $N(y)=N(x)+r$, which is clearly the number of carets in the new source tree for $y$. If $i>N(x)$, then there is no leaf labelled $i$, so we add right carets to the rightmost leaf until there is a leaf labelled $i$. Then attach $r$ carets as before to the leaf $i$. In this case, $N(y)=i+r+1$ since $i>N(x)$.

The proof of $(2)$ is very simple, when one looks at the subdivisions of $[0, \infty)$ given by each tree (see Section 1). Notice that the subdivision corresponding to the target tree gives all intervals of length 1 (except the last one). This last interval has the form $[N(x), \infty)$, and since the diagram is reduced, according to part (i) of Theorem 2.5 of [6], the last two leaves of the source tree do not lie in the same caret. This means that $N(x)$ is the $y$-coordinate of the last breakpoint.

For the case of a general word, not necessarily positive, there is no relation between the $y$-coordinate of the last breakpoint and the distance. The elements

$$
x_{0} x_{1} \ldots x_{k-1} x_{k}^{2} x_{k+1}^{-1} x_{k}^{-1} \ldots x_{1}^{-1} x_{0}^{-1}
$$

have all breakpoints in the square $[0,1] \times[0,1]$, whereas their norm is linear in $k$. But the number of carets in either tree of the reduced diagram is still equivalent to the norm.

Theorem 3. Let $x \in F(2)$ be an element whose normal form is

$$
x_{i_{1}}^{r_{1}} x_{i_{2}}^{r_{2}} \ldots x_{i_{n}}^{r_{n}} x_{j_{m}}^{-s_{m}} \ldots x_{j_{2}}^{-s_{2}} x_{j_{1}}^{-s_{1}}
$$

and let

$$
\begin{aligned}
& y_{1}=x_{i_{1}}^{r_{1}} x_{i_{2}}^{r_{2}} \ldots x_{i_{n}}^{r_{n}}, \\
& y_{2}=x_{j_{1}}^{s_{1}} x_{j_{2}}^{s_{2}} \ldots x_{j_{m}}^{s_{m}}
\end{aligned}
$$

be the two positive words involved in the normal form for $x=y_{1} y_{2}^{-1}$. Then the number $N(x)$ of carets for either tree in the reduced tree diagram for $x$ is equal to the highest number of carets in either tree in the diagrams for $y_{1}$ and $y_{2}$. This 
number of carets is equal to

$$
\begin{aligned}
N(x)= & \max \left\{N\left(y_{1}\right), N\left(y_{2}\right)\right\} \\
= & \max \left\{i_{k}+r_{k}+r_{k+1}+\ldots+r_{n}+1, \text { for } k=1,2, \ldots, n,\right. \\
& \left.j_{l}+s_{l}+s_{l+1}+\ldots+s_{m}+1, \text { for } l=1,2, \ldots, m\right\},
\end{aligned}
$$

and it is equivalent to the distance.

Proof. The equivalence with the distance proceeds as in Proposition 2, except the inequalities are now

$$
\frac{D(x)}{4} \leq N(x) \leq D(x)+1 .
$$

For the number of carets, we only need to realize that to obtain the diagram for $x$ we need to put the two diagrams for $y_{1}$ and $y_{2}$ next to each other with the diagram for $y_{2}$ reversed, and add carets to the one with fewer of them.

The tree diagram obtained will be reduced due to the uniqueness of the normal form, by the results in [6].

Note that the above results only apply to $F(2)$, where for a positive word the number of carets in the source tree and the $y$-coordinate of the last breakpoint coincide. For $F(p)$ with $p>2$, these two numbers are different, but both are still equivalent to the distance. Here are the results for a general $p$, which have straightforward proofs:

Proposition 4. Let

$$
x=x_{i_{1}}^{r_{1}} x_{i_{2}}^{r_{2}} \ldots x_{i_{n}}^{r_{n}}
$$

be a positive word in $F(p)$. Then the number

$$
N_{1}(x)=\max \left\{\left\lfloor\frac{i_{k}}{p-1}\right\rfloor+r_{k}+r_{k+1}+\ldots+r_{n}+1, \text { for } k=1,2, \ldots, n\right\}
$$

is equal to the number of carets in either tree of the reduced $p$-tree diagram for $x$. This number satisfies the inequality

$$
\frac{D(x)}{2(p-1)} \leq N_{1}(x) \leq D(x)+1
$$

The $y$-coordinate of the last breakpoint is in this case:

$$
\begin{array}{r}
N_{2}(x)=\max \left\{i_{k}+r_{k}(p-1)+r_{k+1}(p-1)+\ldots+r_{n}(p-1)+1,\right. \\
\text { for } k=1,2, \ldots, n\} .
\end{array}
$$

As before, it is equivalent to the distance.

For the connection between the number of carets and the word metric for a general word, not necessarily positive, we have

Theorem 5. Let

$$
x=x_{i_{1}}^{r_{1}} x_{i_{2}}^{r_{2}} \ldots x_{i_{n}}^{r_{n}} x_{j_{m}}^{-s_{m}} \ldots x_{j_{2}}^{-s_{2}} x_{j_{1}}^{-s_{1}}
$$


be the unique normal form of an element $x \in F(p)$. Then the number

$$
\begin{array}{r}
N(x)=\max \left\{\left\lfloor\frac{i_{k}}{p-1}\right\rfloor+r_{k}+r_{k+1}+\ldots+r_{n}+1, \text { for } k=1,2, \ldots, n,\right. \\
\left.\left\lfloor\frac{j_{l}}{p-1}\right\rfloor+s_{l}+s_{l+1}+\ldots+s_{m}+1, \text { for } l=1,2, \ldots, m\right\}
\end{array}
$$

is equal to the number of carets in either tree for the reduced diagram for $x$. This number is equivalent to the distance. In particular, it satisfies the inequality

$$
\frac{D(x)}{4(p-1)} \leq N(x) \leq D(x)+1 .
$$

This estimate of the distance in terms of the number of carets in the trees of the diagram will be used extensively in the next sections, to prove that several embeddings of a group $F(p)$ into $F(q)$ are nondistorted.

\section{Embeddings of a Group $F(p)$ into A Group $F(q)$}

There is a large class of embeddings of the groups $F(p)$ that can be understood with the process of caret replacement. Take a $p$-tree diagram of an element of some $F(p)$, and replace each $p$-caret by an arrangement of $q$-carets to obtain the $q$-tree diagram for its image under the embedding. Notice that the diagram obtained after the replacement may need to be reduced. We will only consider replacements where

(1) every $p$-caret is replaced by a fixed arrangement of $q$-carets, or

(2) a $p$-caret is replaced by one of two possible arrangements (both with the same number of $q$-carets) depending on whether the $p$-caret is a right caret or not. The root caret is considered a right caret. In addition we require:

(3) for each of the possible two types of replacement trees, there is a fixed, orderpreserving injection from the leaves of the replaced caret to the leaves of the replacement tree which takes the rightmost leaf to the rightmost leaf.

The above restrictions ensure that the embeddings obtained commute with the group multiplication, yielding group homomorphisms.

As an example, consider the embedding of $F(4)$ into $F(2)$ given by the following replacement of a 4 -caret by an arrangement of 2-carets:
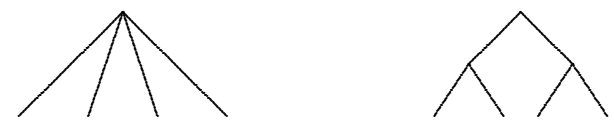

Figure 3. The replacement rule for an embedding of $F(4)$ in $F(2)$

We have the following result:

Theorem 6. An embedding obtained by a caret replacement rule is a quasiisometric embedding.

Proof. Let $f$ be a map of $F(p)$ into $F(q)$ such that every $p$-caret is replaced by an arrangement of $K q$-carets. Then the number of carets obviously satisfies the 

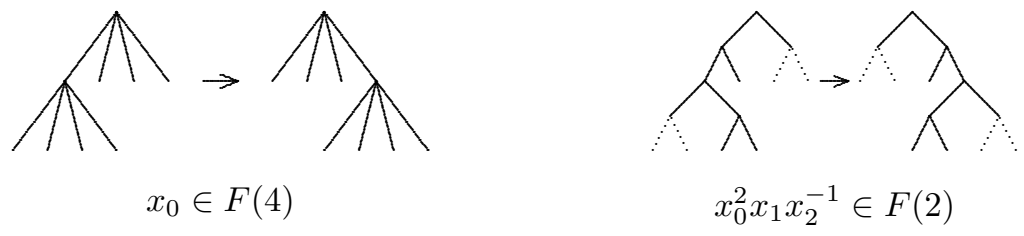

Figure 4 . The image of the generator $x_{0}$ of $F(4)$ in $F(2)$ under the rule of Example 1

following inequality:

$$
N(f(x)) \leq K N(x) .
$$

The number of carets in $f(x)$ is not exactly $K$ times the number of carets in $x$ because the tree diagram obtained after the replacement may not be reduced. But notice that not all the $K q$-carets that come from an individual $p$-caret in $x$ can be reduced, because if this were the case, the original diagram for $x$ would not have been reduced to start with. This yields the inequality

$$
N(x) \leq N(f(x)) .
$$

Since the number of carets is equivalent to the word metric, the latter satisfies two similar inequalities, which prove that the map is a quasi-isometric embedding.

Here is a list of some examples of embeddings that can be obtained this way:

(1) As a generalization of the example of $F(4)$ as a subgroup of $F(2)$, we can embed any $F\left(p^{k}\right)$ into $F(p)$. The rule will just consist of replacing each $p^{k}$ caret by an arrangement of

$$
1+p+p^{2}+\ldots+p^{k-1}=\frac{p^{k}-1}{p-1}
$$

$p$-carets in the following way: start with a $p$-caret and hang another $p$-caret from each leaf. Continue hanging a $p$-caret from each leaf obtained in the previous step until there are $k$ layers of carets.

This embedding occurs naturally when considering the groups as groups of homeomorphisms of the unit interval, where it is clear that any homeomorphism in $F\left(p^{k}\right)$ is also in $F(p)$.

(2) We can also embed $F\left(p^{k}\right)$ into $F(p)$ with a different replacement rule. This time, we will replace all left and interior $p^{k}$-carets by the same arrangement of $p$-carets used in Example 1, but right $p^{k}$-carets are replaced by an arrangement of $1+p+p^{2}+\ldots+p^{k-1}$ right carets, each hanging from the rightmost leaf of the previous one. This rule corresponds to the embedding which arises naturally when the groups are considered as groups of homeomorphisms of the real line, where it is again clear that any homeomorphism in $F\left(p^{k}\right)$ is also in $F(p)$.

The reason the right carets have a different replacement rule is that right carets correspond to the subdivision of an infinite interval, whereas the interior and left carets correspond to subdividing a finite interval.

Note that this gives a different embedding of $F\left(p^{k}\right)$ into $F(p)$ than that of Example 1. 
(3) Assume that $q-1=d(p-1)$. We can embed $F(p)$ into $F(q)$ with the following rule: replace a $p$-caret by a $q$-caret obtained by adding $d-1$ edges between every pair of edges of the $p$-caret. Note that no other caret will hang from the edges that have been added. In other words, we replace each $p$-caret by a $q$ caret, and use the injection which sends leaf $i$ in the $p$-caret to leaf $1+(i-1) d$ in the replacement $q$-caret. Images of the generators in the case $p=2$ and $q=3$ are shown below.

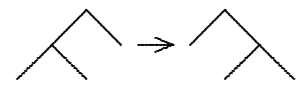

$x_{0} \in F(2)$

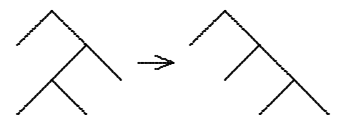

$x_{1} \in F(2)$

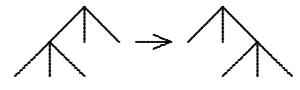

$x_{0} \in F(3)$

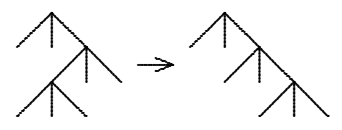

$x_{2} \in F(3)$

FiguRE 5. The two generators of $F(2)$ and their images in $F(3)$

This example has an easy algebraic interpretation: the homomorphism is obtained by sending a generator $x_{i}$ of $F(p)$ to the generator $x_{d i}$ in $F(q)$. The fact that $q-1=d(p-1)$ guarantees that the relations are preserved.

(4) Assume again that $q-1=d(p-1)$. We can embed $F(q)$ into $F(p)$ as follows: replace a right $q$-caret by $d$ right $p$-carets, each hanging from the rightmost leaf of the previous one; and replace each left or interior caret by an arrangement of $d p$-carets each one hanging from the leftmost leaf of the previous one.

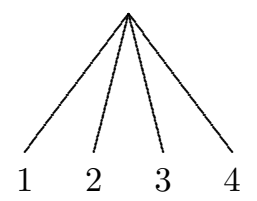

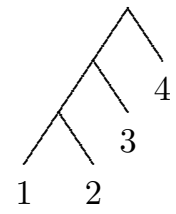

left and interior

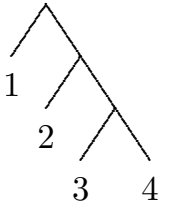

right

Figure 6. The replacement rule for the embedding of $F(4)$ into $F(2)$

The motivation for this choice of replacement rule comes from the connection between the rooted tree diagrams and the normal forms of elements, 
stated in Theorem 2.5 of [6]. The resulting embedding corresponds algebraically to the embedding of $F(q)$ into $F(p)$ which sends a generator $x_{i}$ to $x_{i}^{d}$.

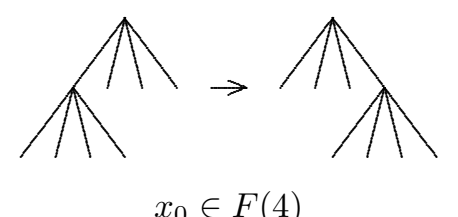

$x_{0} \in F(4)$

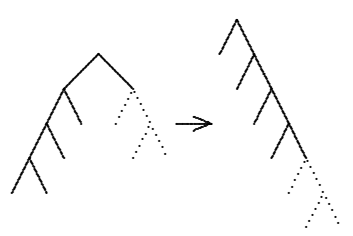

$x_{0}^{3} \in F(2)$

Figure 7 . The image of the generator $x_{0}$ of $F(4)$ in $F(2)$ under the rule in Example 4

(5) Assuming that $p<q$, we can embed $F(p)$ into $F(q)$ in a manner similar to Example 3. For the replacement rule, we add edges to $p$-carets to obtain $q$-carets. By distributing the new edges unevenly through the caret we can obtain many more embeddings. For example, if $p=3$ and $q=6$, we obtain an embedding by adding one edge between the first two edges of a 3 -caret and adding two edges between the other two edges of the 3-caret. In other words, we replace each 3 -caret by a 6 -caret using the injection of the leaves of the 3 -caret into the leaves of the replacement 6 -caret which sends leaf 1 to leaf 1 , leaf 2 to leaf 3, and leaf 3 to leaf 6 . Algebraically, this example corresponds to the embedding which sends the generator $x_{i}$ to the generator $x_{f(i)}$, where $f$ is the function on the natural numbers defined recursively by

$$
\begin{gathered}
f(0)=0, \\
f(1)=2, \\
f(i+2)=f(i)+5 .
\end{gathered}
$$

This example is a particular case of the embeddings stated in Theorem 4.1.2 of [2].

By virtue of Examples 3 and 4, we see that every $F(p)$ can be embedded in $F(2)$, and vice versa, that $F(2)$ can be embedded in any other $F(q)$. This gives the following result:

Theorem 7. Given a pair of integers $p, q \geq 2$, the group $F(p)$ embeds quasiisometrically into $F(q)$.

\section{THE SHIFT MAPS}

Each $F(p)$ admits an interesting group homomorphism to itself. The shift map $\phi_{p}$ for $F(p)$ is defined as the map sending every generator $x_{i}$ in the presentation $\mathcal{P}_{p}$ to $x_{i+1}$. As we have seen before, $\phi_{p}$ satisfies $x_{0}^{-1} \phi_{p}(x) x_{0}=\phi_{p}^{p}(x)$. Of special relevance is the map $\phi_{p}^{p-1}$, because the relators of $\mathcal{P}_{p}$ can be written as

$$
x_{i}^{-1} x_{j} x_{i}=\phi_{p}^{p-1}\left(x_{j}\right), \quad \text { for } i<j ;
$$


so if we take an HNN extension of $F(p)$ by the map $\phi_{p}^{p-1}$, we obtain another copy of $F(p)$.

When the group is seen as a group of homeomorphisms of the real line, the image of an element $x \in F(p)$ by $\phi_{p}^{p-1}$ is the identity in the interval $(-\infty, p-1]$, and in the quadrant $[p-1, \infty) \times[p-1, \infty)$ it consists of a shifted copy of the piece of the graph of $x$ in the quadrant $[0, \infty) \times[0, \infty)$.

Also, it is easy to interpret the maps $\phi_{p}^{p-1}$ in terms of the rooted $p$-tree diagrams. Given a rooted $p$-tree $T$, consider another tree $T^{\prime}$ obtained by taking one single $p$ caret and attaching $T$ by its root to the rightmost vertex of the new caret.

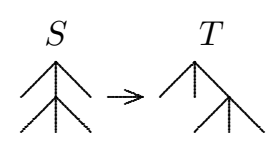

$x_{1} \in F(3)$

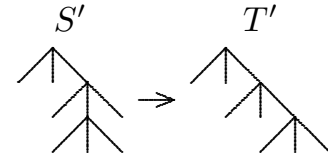

$\phi_{3}^{2}\left(x_{1}\right)=x_{3} \in F(3)$

FIGURE 8. The image of $x_{1} \in F(3)$ under $\phi_{3}^{2}$

Using this construction, it is easy to see that if $(S, T)$ is the reduced tree diagram for $x \in F(p)$, then $\left(S^{\prime}, T^{\prime}\right)$ is the reduced tree diagram for $\phi_{p}^{p-1}(x)$.

Since each of the embeddings in Examples 3, 4 and 5 of the previous section has a simple algebraic formula in terms of the presentation $\mathcal{P}_{p}$, it is trivial to see that they behave nicely with respect to the shift maps. However, using the caret replacement rule, we can see that all of the examples behave well.

Theorem 8. Let $i$ be an embedding of $F(p)$ into $F(q)$ obtained by a caret replacement rule, and let $k$ be the number of right carets in the arrangement used to replace the root (or any right) caret. Then the shift maps satisfy

$$
i \circ \phi_{p}^{p-1}=\phi_{q}^{k(q-1)} \circ i \text {. }
$$

Proof. The proof is straightforward using the interpretation of shift maps in terms of tree diagrams.

\section{REFERENCES}

1. Bieri, R. and Strebel, R., On groups of PL-homeomorphisms of the real line, Notes, Math. Sem. der Univ. Frankfurt, 1985.

2. Brin, M.G. and Guzmán, F., Automorphisms of generalized Thompson groups, J. Algebra 203 (1) (1998), 285-348. MR 99d:20056

3. Brown, K.S., Finiteness properties of groups, J. Pure App. Algebra 44 (1987), 45-75. MR 88m:20110

4. Brown, K.S. and Geoghegan. R., An infinite-dimensional torsion-free $F P_{\infty}$ group, Invent. Math. 77 (1984), 367-381. MR 85m:20073

5. Burillo, J., Quasi-isometrically embedded subgroups of Thompson's group F, J. Algebra 212 (1999), 65-78. MR 99m:20051

6. Cannon, J.W., Floyd, W.J., and Parry, W.R., Introductory notes on Richard Thompson's groups, Enseign. Math. 42 (1996), 215-256. MR 98g:20058

7. Cleary, S., Groups of piecewise-linear homeomorphisms with irrational slopes, Rocky Mountain J. Math. 25 (1995), 935-955. MR 97d:20040

8. Fordham, S.B., Minimal Length Elements of Thompson's Group F, Thesis, Brigham Young University, 1995.

9. Guba, V. and Sapir, M., Diagram Groups, Mem. Amer. Math. Soc. 130 (1997). MR 98f:20013 
10. Higman, G., Finitely presented infinite simple groups, Notes on Pure Math., vol. 8, Australian National University, Canberra, 1974. MR 51:13049

11. Stein, M., Groups of piecewise linear homeomorphisms, Trans. Amer. Math. Soc. 332 (2) (1992), 477-514. MR 92k:20075

Departament de Matemàtiques, Universitat Autònoma de Barcelona, 08192 BarceLONA, SPAIN

Current address: Department of Applied Mathematics, University Politecnica de Catalunya, Campus Nord, Jordi Girona 1, 08034 Barcelona, Spain

E-mail address: burillo@mat.upc.es

Department of Mathematics, City College of CUNy, New York, New York 10031

E-mail address: cleary@matho.sci.ccny.cuny.edu

Department of Mathematics, Trinity College, Hartford, Connecticut 06106

E-mail address: mstein@mail.trincoll.edu 